\title{
Experimental Evidence on the Mechanism of Dissociative Electron Attachment to Formic Acid
}

\author{
R. Janečková, D. Kubala, O. May, J. Fedor, and M. Allan \\ Department of Chemistry, University of Fribourg, Chemin du Musée 9, 1700 Fribourg, Switzerland
}

(Received 13 August 2013; published 20 November 2013)

\begin{abstract}
Two mechanisms for dissociative electron attachment in $\mathrm{HCOOH}$, the formation of $\mathrm{HCOO}^{-}+\mathrm{H}$, were proposed in the literature: (i) via a direct electron attachment into a $\sigma^{*}$ resonance, augmented by dipole binding of the incident electron [G. A. Gallup et al., Phys. Rev. A 79, 042701 (2009)], and (ii) with the $1.8 \mathrm{eV} \pi^{*}$ resonance as a doorway state, linked to the products by symmetry lowering-distortion of the temporary anion, primarily the $\mathrm{C}-\mathrm{H}$ bond, from the planar symmetry [T. N. Rescigno et al., Phys. Rev. Lett. 96, 213201 (2006)]. The later mechanism implies a reduction of the cross section upon deuteration of the hydrogen bonded to the $\mathrm{C}$ atom, whereas the former mechanism would leave the cross section unaffected. Our experimental absolute cross sections for the four isotopomers of formic acid show that deuteration on the $\mathrm{C}$ atom reduces the cross section value only marginally (by $12 \%$ ) compared to deuteration on the $\mathrm{O}$ atom (reduction by a factor of 16), and thus favor mechanism (i).
\end{abstract}

DOI: 10.1103/PhysRevLett.111.213201

PACS numbers: $34.80 . \mathrm{Ht}$

Numerous applications of electron-induced chemical change, both in the gas phase-in plasmas [1], in outer space, and in planetary and comet atmospheres-and in the condensed phase - in radiation therapy and in the focused electron-beam-induced processing nanofabrication technique [2] - justify the quest for understanding the mechanism of dissociative electron attachment (DEA). This effort is more complicated than the closely related quest for theoretical understanding of photochemistry, because the ground and excited states of the temporary negative ion mediating the DEA are generally resonances, i.e., are subject to spontaneous electron loss (autodetachment), a decay channel competing with dissociation. Autodetachment occurs on a time scale comparable or faster than dissociation, thus complicating both the calculation of the resonant potential surfaces and the dissociation dynamics. An additional complication is the prevalence of nonadiabatic and nonlocal phenomena.

This Letter presents experimental evidence for the mechanism of the loss of a hydrogen atom in DEA to formic acid at low energy - which may be considered as a prototype for this reaction in a number of compounds, including biomolecules [3].

Generally, two qualitatively different mechanisms are found for the loss of a hydrogen atom in DEA at low energies:

(i) Compounds with double or triple bonds. This class of compounds has pronounced low-lying $(0-5 \mathrm{eV}) \pi^{*}$ shape resonances, revealed experimentally as relatively narrow bands in cross sections for vibrational excitation (VE) [4]. Acetylene $\mathrm{C}_{2} \mathrm{H}_{2}$ is a suitable prototype with a $\pi_{g}^{*}$ shape resonance at $3.6 \mathrm{eV}$. Loss of a hydrogen atom (production of $\mathrm{C}_{2} \mathrm{H}^{-}$), clearly mediated by the $\pi_{g}^{*}$ resonance, is observed in DEA. The mechanism was revealed by the $a b$ initio calculations of the absolute DEA cross sections of Chourou and Orel [5,6], which involved time-dependent calculation of the evolution of the nuclear wave packet on the resonant potential surface, represented by a local complex potential obtained by the $a b$ initio complex Kohn method. An innovative feature of this work was taking several dimensions of the potential surface explicitly into account. This is essential because the mechanism was shown to be indirect. The $\pi^{*}$ resonance, with its relatively narrow autodetachment width, acts as a doorway, and a substantial distortion of the molecular framework (symmetry lowering) is required to bypass an energy barrier on the way of dissociation. The theoretical results were validated by a favorable comparison with experimental cross sections, both in terms of the absolute values [7] and the isotope effect [8]. The symmetry lowering pathway prevails also in propyne $\mathrm{CH}_{3} \mathrm{C} \equiv \mathrm{C}-\mathrm{H}$ where the acetylenic $\mathrm{C}-\mathrm{H}$ bond was found to break, although the methyl group $\mathrm{CH}_{2}-\mathrm{H}$ bond could break without symmetry lowering [9]. The symmetry-lowering mechanism is common among compounds with $\pi$ bonds; two other important examples are vinyl chloride and chlorobenzene [10,11].

(ii) Compounds without multiple bonds. $\pi^{*}$ resonances are not essential for hydrogen loss at low energies, however, as shown by compounds with only single bonds, for example hydrogen halides [12,13], but also by polyatomic molecules like alcohols [14]. Experimental signatures of this mechanism are peaks at threshold and, in the case of halogen halides, downward steps at thresholds for vibrational excitation $[15,16]$. The assignment to $\sigma^{*}$ shape resonances is more problematic in this case than the assignment to $\pi^{*}$ resonances in the above cases, because the VE cross sections do not clearly reveal $\sigma^{*}$ resonances as bands in the spectra; instead they have sharp narrow "threshold peaks" assigned to virtual states and vibrational Feshbach resonances [17]. Two theoretical approaches succeeded to quantitatively calculate the DEA cross sections, including the isotope effect and the temperature dependence, for 
(diatomic) molecules without double bonds. They are the nonlocal resonance theory and the effective range and modified effective range $R$-matrix theory (see, for example, Refs. [12,18-20]; see Ref. [21] for the comparison of the two methods). Both approaches have in common that they include nonlocal effects and nonadiabatic processes, which are essential in the $\sigma^{*}$ cases and which are not included in the local complex potential method. The resonance $R$-matrix theory was recently applied to polyatomic molecules [22-24], which were treated as pseudodiatomics; the multidimensionality was not explicitly taken into account.

An open question arises in molecules like formic acid, which have both a $\pi^{*}$ resonance like acetylene and a polar $\sigma$ bond like $\mathrm{HBr}$, so that both the "HBr-like" and the "acetylenelike" mechanisms could be applicable. Both were proposed in the literature. Rescigno et al. [25] calculated an ab initio $\pi^{*}$ resonance potential surface using the complex Kohn method and found that, as in the acetylene case, the dissociation in planar geometry is hindered by an energy barrier that can be by-passed by distortion of the molecular framework out of planarity. Gallup et al. [26] calculated the cross section under the assumption of the $\mathrm{HBr}$-like mechanism, a $\sigma^{*}$ electron attachment without involvement of the $\pi^{*}$ resonance, and obtained an absolute cross section that agreed well in terms of shape and the presence of the $\mathrm{HBr}$-like downward steps with the experiment [27]. A discussion developed subsequently in the literature $[28,29]$. In this work we present experimental evidence on which of the two mechanisms is operative, derived from absolute DEA cross sections of deuterated isotopomers of formic acid.

Experiment.-We measured absolute cross sections with a "quantitative" time-of-flight instrument [8]. It is optimized for collection efficiency independent of ion mass and initial kinetic energy, although this capacity is not essential in the present work where exclusively the formate anion isotopomers with nearly the same mass and with nearly zero kinetic energy are measured.

The absolute calibration of the cross section was against the $4.4 \mathrm{eV}$ band of $\mathrm{O}^{-}$production from $\mathrm{CO}_{2}$. The $\mathrm{HCOO}^{-}$ band is relatively narrow, with a sharp peak; the apparent peak cross section thus depends on instrumental resolution. We therefore calibrated our spectra to the energyintegrated cross section-the area under the DEA band. We used the value of $13.3 \mathrm{pm}^{2} \mathrm{eV}$, an average of our own measurement [8] and earlier data [30]. This value is consistent with that used to normalize our previous work, for example Refs. [8,9]. The error of the absolute measurement ( 2 standard deviations) is taken as $\pm 20 \%$ and it includes the $\pm 15 \%$ error of the reference $\mathrm{O}^{-}$from $\mathrm{CO}_{2}$ value. The relative values of the individual isotopomers are more reliable, within $\pm 5 \%$ ( 1 standard deviation).

We then measured the DEA band under higher resolution with a trochoidal electron spectrometer combined with
TABLE I. Peak and integrated DEA cross sections $\left(\mathrm{pm}^{2}\right.$ and $\mathrm{pm}^{2} \mathrm{eV}$, respectively).

\begin{tabular}{lcccc}
\hline \hline Target & HCOOH & DCOOH & HCOOD & DCOOD \\
\hline Peak CS & 156 & 142 & 11.6 & 8.68 \\
Integrated CS & 98.4 & 86.2 & 6.25 & 5.45 \\
\hline \hline
\end{tabular}

a quadrupole mass filter [31]. The energy width, judged from the electron beam retardation curve, was about $45 \mathrm{meV}$ at a beam current of about $2 \mathrm{nA}$. The sample gas temperature was $60^{\circ} \mathrm{C}$ in both instruments. These spectra were normalized to the time-of-flight absolute values in a way as to conserve the areas under the bands. The resulting peak cross section for $\mathrm{HCOOH}, 156 \pm 30 \mathrm{pm}^{2}$ (see Table I), compares favorably with the value of $170 \pm$ $60 \mathrm{pm}^{2}$ of Pelc et al. [27] and $140 \pm 60 \mathrm{pm}^{2}$ of Prabhudesai et al. [32].

A long passivation period (several days) is required when switching isotopomers because deuterium is initially replaced by hydrogen, by exchange with compounds (presumably mainly $\mathrm{H}_{2} \mathrm{O}$ ) adsorbed on the inner surfaces of the gas inlet system. The passivation process was monitored by a residual gas analyzer attached to the instrument. The passivation is particularly important for the OD compounds. A small HCOOD impurity in $\mathrm{HCOOH}$ does not perturb the result substantially, because HCOOD has a small cross section, but a small $\mathrm{HCOOH}$ impurity in HCOOD, with its much larger cross section, does perturb the result substantially.

Results and discussion.-The work of Rescigno et al. [25] indicates that the critical distortion of the nuclear framework connecting the $\pi^{*}$ resonance to the product $\mathrm{H}$ atom and $\mathrm{HCOO}^{-}$formate anion (both with $\sigma$ symmetry) is a departure of the $\mathrm{H}$ atom of the $\mathrm{C}-\mathrm{H}$ bond from the plane of the molecule, as shown schematically in Fig. 1. This may seem surprising because this hydrogen is not dissociating and is relatively far from the $\mathrm{O}-\mathrm{H}$ bond. This distortion perturbs the up-down symmetry of the $\pi^{*}$ orbital, however, whose nodal surface then no longer passes through the $\mathrm{O}-\mathrm{H}$ bond, i.e., permits conjugation of the $\pi^{*}$ and the $\mathrm{O}-\mathrm{H} \sigma^{*}$ orbitals, leading to dissociation. If this $\mathrm{C}-\mathrm{H}$ out-of-plane distortion is crucial, then the DEA cross section should drop substantially upon deuteration of the

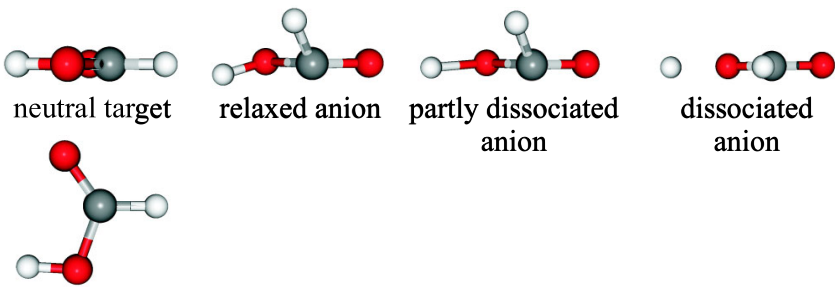

FIG. 1 (color online). Bottom: the structure of neutral formic acid. Top: qualitative structures, seen in the plane of the molecule, of the neutral molecule (left), the relaxed negative ion, and the negative ion with progressively stretched $\mathrm{O}-\mathrm{H}$ bond. 
C-bonded hydrogen because this distortion is in competition with fast autodetachment and the slower motion of deuterium as compared to hydrogen will shift this competition in favor of autodetachment. The effect should be large, because the relatively small magnitude of the DEA cross section indicates that already in the nondeuterated compound the competition is largely on the autodetachment side and the cross section must be very sensitive to slowing down of the dissociation dynamics. (As an example, the isotope effect in acetylene is a factor of 14.4 [8].)

Measurements of the cross sections of the C-deuterated formic acids DCOOH and DCOOD is thus a way of experimentally determining whether the $\mathrm{C}-\mathrm{H}$ out-ofplane excursion is critical for DEA. The experimental results in Fig. 2 and Table I show that this is not the case to any substantial degree. This finding thus favors the $\mathrm{HBr}$-like or $\sigma^{*}$ mechanism as proposed by Gallup et al. [26].

A number of remarks can be added to this finding. An $a b$ initio calculation of DEA to $\mathrm{HCN}[33,34]$ (validated experimentally [35]) led to the surprising finding that although the electronic structure is the same as in the case of acetylene, and also the potential surfaces of the two anions are similar (i.e., an energy barrier is

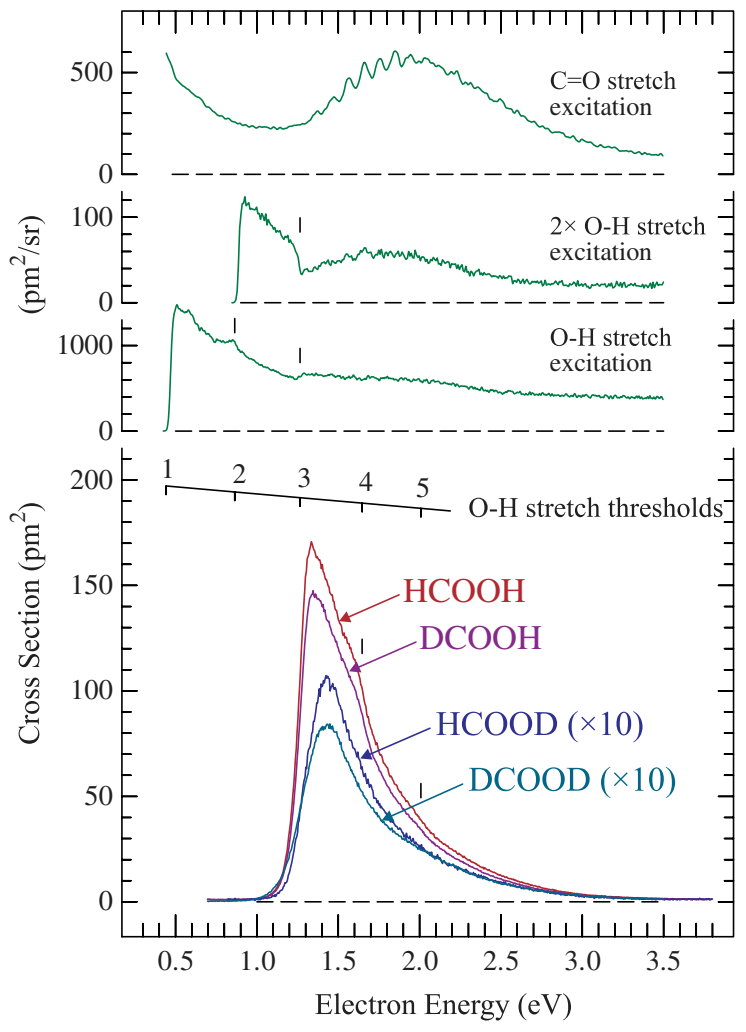

FIG. 2 (color online). Bottom: cross sections for production of the formate anion shown as a function of electron energy. Note that the cross sections for the $\mathrm{O}-\mathrm{D}$ deuterated compounds are shown $10 \times$ vertically expanded. Top: cross sections for vibrational excitation, revealing shape resonances in formic acid (from Ref. [36]). encountered for dissociation in linear geometry but may be by-passed by bending the $\pi^{*}$ anion out of linearity), a dynamics calculation revealed that the hydrogen prefers to tunnel through the barrier rather than traveling around it in $\mathrm{HCN}$. This could also happen for the $\mathrm{O}-\mathrm{H}$ hydrogen in formic acid; the dissociation of the $\pi^{*}$ resonance would then proceed without involvement of the $\mathrm{C}-\mathrm{H}$ hydrogen and would also explain the present experimental finding.

The measured isotope effect for deuteration on the $\mathrm{O}$ atom is a factor of 16 for both $\mathrm{HCOOH}$ and $\mathrm{DCOOH}$. The strong effect is easily understandable as a consequence of the slower motion of the heavier deuterium and thus a less favorable competition of dissociation over autodetachment. The isotope effect for deuteration on the $\mathrm{C}$ atom ( $12.5 \%$ for both $\mathrm{HCOOH}$ and HCOOD) is much weaker but likely to be significant.

Nuclear relaxation during the lifetime of a given resonance leads, apart from DEA, also to vibrational excitation and the vibrational excitation cross sections consequently provide a convenient means of detecting resonances in formic acid [36,37]. Three representative cross sections are shown in the top part of Fig. 2. The top curve shows the cross section for exciting the $\mathrm{C}=\mathrm{O}$ stretch vibration and visualizes the $\pi^{*}$ resonance, antibonding with respect to the $\mathrm{C}=\mathrm{O}$ bond. The shape of the band, with only a very gradual onset, indicates that the equilibrium geometry of the anion is very different from that of the neutral molecule. The vertical attachment is around $1.8 \mathrm{eV}$. The adiabatic attachment energy is below the DEA threshold, so that DEA via the $\pi^{*}$ resonance is possible energetically, although it is not favored by the small Franck-Condon factor.

The $1.8 \mathrm{eV} \pi^{*}$ resonance is, not surprisingly, barely visible in the $\mathrm{O}-\mathrm{H}$ stretch excitation cross sections because the $\pi^{*}$ orbital is not antibonding with respect to the $\mathrm{O}-\mathrm{H}$ bond. The general shapes of the two $\mathrm{O}-\mathrm{H}$ stretch excitation curves resemble those of halogen hydrides, for example $\mathrm{HBr}[12,17]$, with a threshold peak and cusps at vibrational thresholds. This suggests that a similar physical mechanism is involved. The magnitude of the VE cross section is large, revealing a resonant enhancement, but the assignment of this enhancement to a $\sigma^{*}$ resonance is less clear-cut than in the case of the $\pi^{*}$ resonance in the $\mathrm{C}=\mathrm{O}$ stretch cross section; no obvious band is observed. We therefore suggest that the situation is more complex, similar to $\mathrm{HBr}$, and that a valence $\sigma^{*}$ state but also dipole binding and nonlocal phenomena are involved. This conclusion may be related to the fact that the $\sigma^{*}$ resonance does not appear in ab initio scattering calculations [28]. (There is an interesting detail, however-the threshold peak is wider in the formic acid $\mathrm{O}-\mathrm{H}$ stretch excitation than in $\mathrm{HBr}$.)

The DEA threshold energy for $\mathrm{HCOOH}$, derived from the gas phase acidity at $0 \mathrm{~K}, \Delta_{\text {acid }} H_{0}=345.2 \pm$ $0.5 \mathrm{kcal} / \mathrm{mol}$ [38], is $E_{\mathrm{th}}=1.36 \pm 0.02 \mathrm{eV}$. This value is higher than that derived from our spectra, $1.29 \mathrm{eV}$. We thus 
recommend that the gas phase acidity at $0 \mathrm{~K}$ is reduced to $\Delta_{\text {acid }} H_{0}=343.5 \mathrm{kcal} / \mathrm{mol}$. The threshold values for HCOOD and DCOOD are higher because of the differences of the zero point energies of the target and the products, and this is born out by the higher peak values for the HCOOD and DCOOD bands. Becke threeparameter Lee-Yang-Parr hybrid functional calculations of zero point energies indicate that the threshold should be $92 \mathrm{meV}$ higher in HCOOD than in $\mathrm{HCOOH}$, in agreement with the observation.

The onsets of the HCOOD and DCOOD DEA bands are more gradual than those of $\mathrm{HCOOH}$ and $\mathrm{DCOOH}$. The cause could be hot bands, which are expected to be stronger for the two compounds deuterated on the $\mathrm{O}$ atom, with their smaller absolute DEA cross sections. The prototype of this behavior is $\mathrm{HCl}$ [39].

An important feature of the DEA bands in formic acid is the downward steps [27] resembling those found in $\mathrm{HBr}$. They are due to competition of the DEA and VE channels and occur whenever a new vibrational excitation channel opens. We enhanced the visibility of these steps by subtracting a smooth background from the DEA bands as shown in Fig. 3. (The energies of the $\mathrm{O}-\mathrm{H}$ stretch overtones are taken from Refs. [40,41].) Steps at the thresholds for four and five quanta of $\mathrm{O}-\mathrm{H}$ stretch excitation are visible for both $\mathrm{HCOOH}$ and $\mathrm{DCOOH}$. Two more steps are discernible just above threshold and assigned to the openings of the ( $3 \mathrm{O}-\mathrm{H}$ stretch $+\mathrm{C}=\mathrm{O}$ stretch) and the (3 O-H stretch $+\mathrm{O}-\mathrm{C}-\mathrm{O}$ deformation) excitation

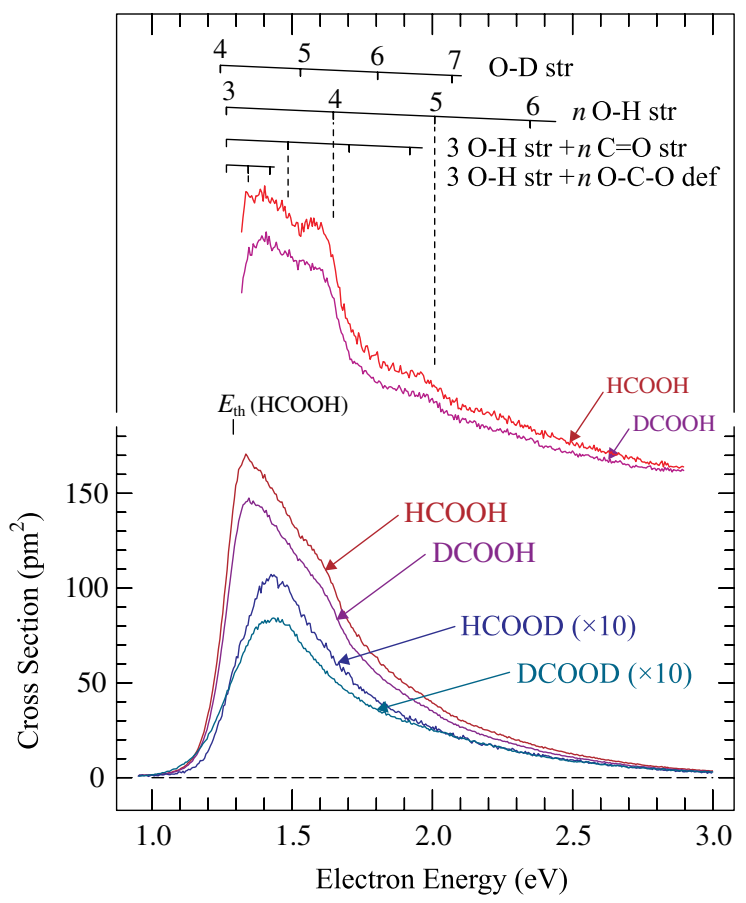

FIG. 3 (color online). Bottom: Cross sections for production of the formate anion shown as a function of electron energy. Top: The cross sections with a smooth background subtracted to enhance the steplike structures. channels. Note that the cross sections for both the $\mathrm{O}-\mathrm{C}-\mathrm{O}$ deformation and $\mathrm{C}=\mathrm{O}$ stretch vibrations have intense threshold peaks [36] and would thus be expected to cause downward steps in the DEA cross section. The appearance of the $\mathrm{C}=\mathrm{O}$ stretch and $\mathrm{O}-\mathrm{C}-\mathrm{O}$ deformation-related structure demonstrates the inherent multidimensionality of the process.

Conclusions. - The primary conclusion of our study is experimental: the isotope effect for deuteration of the $\mathrm{O}-\mathrm{H}$ hydrogen is about $100 \times$ larger than that for deuteration of the $\mathrm{C}-\mathrm{H}$ hydrogen. In view of the fact that the nuclear motion competes with very fast autodetachment this means that the reaction path of the dissociative electron attachment does not involve any significant motion of the $\mathrm{C}-\mathrm{H}$ hydrogen. The $a b$ initio scattering calculation of Rescigno et al. [25] revealed that the $\pi^{*}$ resonance cannot dissociate directly, that a $\pi^{*}-\sigma^{*}$ coupling must first be induced by a deformation of the molecular frame, and that the relevant deformation is the out-of-plane excursion of the $\mathrm{C}-\mathrm{H}$ hydrogen. This excursion would imply a large isotope effect for deuteration of the $\mathrm{C}-\mathrm{H}$ hydrogen, in contrast with our observation. The combination of the present experimental result with the theoretical result of Rescigno et al. [25] thus indicates that the " $\pi^{*}$ mechanism" makes only a minor contribution to the observed cross section. Part of the reason why the contribution is weak may be that the DEA occurs at an energy below the center of the $\pi^{*}$ resonance, at the edge of the FranckCondon range for $\pi^{*}$ attachment.

The " $\sigma^{*}$ mechanism" proposed by Gallup et al. [26] does not require a $\mathrm{C}-\mathrm{H}$ out-of-plane bending and is thus compatible with the present results. In addition, the downward steps at the thresholds for the excitation of the $4 \mathrm{O}-\mathrm{H}$ stretch and $5 \mathrm{O}-\mathrm{H}$ stretch vibrational states, predicted by this theory, are clearly observed, and the measured peak cross section value for $\mathrm{HCOOH}$ agrees very well with the calculation.

A minor conclusion is drawn from the weaker structures in the DEA cross section, assigned as downward steps at the thresholds for the excitation of the $(3 \mathrm{O}-\mathrm{H}$ stretch $+1 \mathrm{C}=\mathrm{O}$ stretch) and $(3 \mathrm{O}-\mathrm{H}$ stretch + $1 \mathrm{O}-\mathrm{C}-\mathrm{O}$ deformation) combination vibrations. They point out that the DEA process has some multidimensional character, although the success of the unidimensional theory indicates that the $\mathrm{O}-\mathrm{H}$ stretch dimension dominates by far. A second indication of a multidimensional character is the weak but significant isotope effect for deuteration on the $\mathrm{C}$ atom. The ultimate goal would be a unified theoretical treatment that includes both the nonlocal effects and dipole binding, and the multidimensional phenomena.

This research is part of projects No. 200020-131962/1 and No. PZ00P2_132357 of the Swiss National Science Foundation, of project SBF No. C07.0018 of the State Secretariat for Education and Research related to COST Action CM0601, and of COST Action CM1301. 
[1] A. Agarwal and M. J. Kushner, J. Vac. Sci. Technol. A 27, 37 (2009).

[2] I. Utke, P. Hoffmann, and J. Melngailis, J. Vac. Sci. Technol. B 26, 1197 (2008).

[3] S. Ptasinska, S. Denifl, P. Scheier, E. Illenberger, and T. D. Märk, Angew. Chem., Int. Ed. Engl. 44, 6941 (2005).

[4] G. J. Schulz, Rev. Mod. Phys. 45, 423 (1973).

[5] S. T. Chourou and A.E. Orel, Phys. Rev. A 77, 042709 (2008).

[6] S. T. Chourou and A.E. Orel, Phys. Rev. A 80, 034701 (2009).

[7] O. May, J. Fedor, B. C. Ibănescu, and M. Allan, Phys. Rev. A 77, 040701(R) (2008).

[8] O. May, J. Fedor, and M. Allan, Phys. Rev. A 80, 012706 (2009).

[9] R. Janečková, O. May, and J. Fedor, Phys. Rev. A 86, 052702 (2012).

[10] P. D. Burrow, A. Modelli, S. C. Chiu, and K. D. Jordan, Chem. Phys. Lett. 82, 270 (1981).

[11] K. L. Stricklett, S. C. Chu, and P. D. Burrow, Chem. Phys. Lett. 131, 279 (1986).

[12] M. Č́ižek, J. Horáček, A.-C. Sergenton, D. B. Popović, M. Allan, W. Domcke, T. Leininger, and F. X. Gadea, Phys. Rev. A 63, 062710 (2001).

[13] J. Fedor, O. May, and M. Allan, Phys. Rev. A 78, 032701 (2008).

[14] B. C. Ibănescu, O. May, A. Monney, and M. Allan, Phys. Chem. Chem. Phys. 9, 3163 (2007).

[15] J. P. Ziesel, I. Nenner, and G. J. Schulz, J. Chem. Phys. 63, 1943 (1975).

[16] R. Abouaf and D. Teillet-Billy, J. Phys. B 10, 2261 (1977).

[17] H. Hotop, M.-W. Ruf, M. Allan, and I. I. Fabrikant, Adv. At. Mol. Opt. Phys. 49, 85 (2003).

[18] W. Domcke, Phys. Rep. 208, 97 (1991).

[19] I. I. Fabrikant, S. A. Kalin, and A. K. Kazansky, J. Chem. Phys. 95, 4966 (1991).

[20] D. Teillet-Billy and J.P. Gauyacq, J. Phys. B 17, 4041 (1984).
[21] I. I. Fabrikant, Comments At. Mol. Phys. 24, 37 (1990).

[22] A. Schramm, I. I. Fabrikant, J. M. Weber, E. Leber, M.-W. Ruf, and H. Hotop, J. Phys. B 32, 2153 (1999).

[23] I. I. Fabrikant, J. Phys. Conf. Ser. 204, 012004 (2010).

[24] G. A. Gallup and I. I. Fabrikant, Phys. Rev. A 83, 012706 (2011).

[25] T. N. Rescigno, C. S. Trevisan, and A. E. Orel, Phys. Rev. Lett. 96, 213201 (2006).

[26] G. A. Gallup, P. D. Burrow, and I. I. Fabrikant, Phys. Rev. A 79, 042701 (2009).

[27] A. Pelc, W. Sailer, P. Scheier, and T. D. Märk, Vacuum 78, 631 (2005).

[28] T. N. Rescigno, C. S. Trevisan, and A. E. Orel, Phys. Rev. A 80, 046701 (2009).

[29] G. A. Gallup, P. D. Burrow, and I. I. Fabrikant, Phys. Rev. A 80, 046702 (2009).

[30] Y. Itikawa, J. Phys. Chem. Ref. Data 31, 749 (2002).

[31] M. Stepanović, Y. Pariat, and M. Allan, J. Chem. Phys. 110, 11376 (1999).

[32] V. S. Prabhudesaia, D. Nandia, A. H. Kelkara, R. Parajulib, and E. Krishnakumar, Chem. Phys. Lett. 405, 172 (2005).

[33] S. T. Chourou and A.E. Orel, Phys. Rev. A 80, 032709 (2009).

[34] S. T. Chourou and A.E. Orel, Phys. Rev. A 83, 032709 (2011).

[35] O. May, D. Kubala, and M. Allan, Phys. Rev. A 82, 010701 (2010).

[36] M. Allan, J. Phys. B 39, 2939 (2006).

[37] M. Allan, Phys. Rev. Lett. 98, 123201 (2007).

[38] N. Eyet, S. M. Villano, and V. M. Bierbaum, Int. J. Mass Spectrom. 283, 26 (2009).

[39] M. Allan and S. F. Wong, J. Chem. Phys. 74, 1687 (1981).

[40] D. L. Howard and H. G. Kjaergaarda, J. Chem. Phys. 121, 136 (2004).

[41] K. Marushkevich, L. Khriachtchev, J. Lundell, A. V. Domanskaya, and M. Räsänen, J. Mol. Spectrosc. 259, 105 (2010). 